Anestesiología

Enero-Marzo 2022

Vol. 45. No. 1. pp 16-22

doi: $10.35366 / 102898$

\section{Comportamiento de presiones intrapulmonares con inversión de la relación inspiración espiración en colecistectomía laparoscópica}

\author{
Behavior of intrapulmonary pressures with inversion of the \\ inspiration-expiration ratio in laparoscopic cholecystectomy
}

\author{
Dra. Lilian Liset González-Bermejo,* Dr. José Antonio Pozo-Romero, ${ }^{\ddagger}$ \\ Dra. Mayda Correa-Borrell,,$^{\S}$ Dr. Carlos de la Paz-Estrada ${ }^{\llbracket}$
}

Citar como: González-Bermejo LL, Pozo-Romero JA, Correa-Borrell M, de la Paz-Estrada C. Comportamiento de presiones intrapulmonares con inversión de la relación inspiración espiración en colecistectomía laparoscópica. Rev Mex Anestesiol. 2022; 45 (1): 16-22. https://dx.doi.org/10.35366/102898

RESUMEN. Objetivo: Evaluar el comportamiento de las presiones intrapulmonares con la inversión del índice inspiración espiración durante la colecistectomía electiva videolaparoscópica. Material y métodos: Se realizó un ensayo clínico controlado fase tres acerca de tales presiones intrapulmonares, durante operaciones de colecistectomía laparoscópica, en el Hospital Universitario «Manuel Ascunce Domenech», en el período de septiembre del 2016 a febrero del 2020. El universo estuvo constituido por los pacientes que cumplieron con los criterios de inclusión, y una muestra de 106 de ellos, los cuales se dividieron en dos conjuntos de 53 personas cada uno, por método aleatorio simple. La fuente primaria de obtención de datos la constituyeron las historias clínicas y el resultado de una encuesta. Los datos se recogieron en un modelo diseñado en correspondencia con la bibliografía. Resultados: Se presentó aumentos de la presión inspiratoria pico y presión meseta luego del neumoperitoneo, con descenso de ambas tras la transposición del índice I:E, además de hipertensión arterial, arritmias e hipotensión arterial, sin guardar relación con la aplicación del indicador I:E invertido. Conclusiones: La inversión de la inspiración espiración contribuyó a mejorar los efectos del neumoperitoneo sobre dichas presiones intrapulmonares, manteniendo una oxigenación adecuada y nulos efectos cardiovasculares.

ABSTRACT. Objective: Evaluating the behavior of intrapulmonary pressures with the inversion of the inspiration expiration index, during elective videolaparoscopic cholecystectomy. Material and methods: A phase three controlled clinical trial was conducted on intrapulmonary pressures during laparoscopic cholecystectomies at the «Manuel Ascunce Domenech» University Hospital, from September 2016 to February 2020, the universe consisted of patients who met the inclusion criteria, from which a sample of 106 patients was selected, by a simple randomizing method. The primary source of data collection was the medical records and the result of a survey. These data were collected in a model designed in correspondence with the bibliography reviewed. Results: Measured by variation in peak inspiratory pressure and plateau pressure after pneumoperitoneum, with significant decrease of both after inversion of $I$ :E index. More than half of the patients presented arterial hypertension, arrhythmias and arterial hypotension, without them being related to the application of the inverted I:E index. Conclusions: The inversion of inspiratory expiration contributed to improve the effects of pneumoperitoneum on intrapulmonary pressures, with adequate oxygenation and no cardiovascular effects.

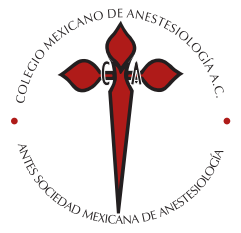

Palabras clave: Colecistectomía videolaparoscópica, inversión índice inspiración-espiración, neumoperitoneo.

Keywords:

Videolaparoscopic

cholecystectomy, inversion index inspiratory expiration, pneumoperitoneum.

* Especialista de primer grado en anestesiología y reanimación. Profesor instructor. Hospital Pediátrico Provincial «Ignacio Agramonte Piña». Camagüey, Cuba.

$\ddagger$ Especialista de segundo grado en anestesiología y reanimación. Máster en urgencias médicas. Profesor asistente. Hospital Universitario «Manuel Ascunce Domenech». Camagüey, Cuba. $\S$ Especialista de segundo grado en anestesiología y reanimación. Máster en urgencias médicas. Investigadora auxiliar. Profesor auxiliar y consultante. Hospital Universitario «Manuel Ascunce Domenech». Camagüey, Cuba. ^ Especialista de segundo grado en anestesiología y reanimación. Máster en urgencias médicas. Profesor asistente. Hospital Pediátrico Provincial «Ignacio Agramonte Piña». Camagüey, Cuba.

Correspondencia:

Carlos de la Paz-Estrada

E-mail: carlosdelapazestrada@ gmail.com

Recibido: 23-01-2020

Aceptado: 26-11-2020

\section{INTRODUCCIÓN}

L a cirugía laparoscópica se inició a comienzos del siglo XX, y a partir de la década de 1950 ha sido tal su despliegue que hoy prácticamente todos los procedimientos ginecológicos pueden realizarse por este medio y la mayoría de ellos de manera ambulatoria.
En Cuba se inició en la ciudad de la Habana en el año 1991, y en Camagüey en el año 1996, en el Hospital Provincial «Manuel Ascunce Domenech», siendo sus propulsores el Dr. Israel González Moya como cirujano, y la Dra. Mayda Correa Borrel como anestesióloga.

La cirugía de mínimo acceso representa un enorme avance tecnológico, que ha tenido una gran aceptación entre los 
cirujanos con respecto a la cirugía abierta, ya que proporciona un considerable número de ventajas a los pacientes que son tributarios a la misma, por ejemplo el menor dolor postoperatorio asociado a una herida más pequeña, la menor aparición de íleo paralítico postoperatorio, la menor estadía hospitalaria y la incorporación más rápida a la vida social y laboral, entre otras ${ }^{(1)}$.

A fin de realizar la intervención, el neumoperitoneo practicado requiere de bióxido de carbono como gas para éste, y el uso de posiciones antifisiológicas durante el acto quirúrgico influye de manera significativa en la mayoría de los órganos y sistemas, causando alteraciones fisiopatológicas fundamentalmente en los sistemas respiratorio y cardiovascular, que de no ser manejadas adecuadamente por el anestesiólogo podrían dar lugar a la aparición de complicaciones. La repercusión fisiopatológica secundaria al aumento de la presión intraabdominal y a las posturas que adopta el paciente cobra mayor importancia en dependencia de cuán deficiente sea el estado preoperatorio de los aparatos cardiovascular y respiratorio del paciente, al igual que la complejidad y duración del procedimiento quirúrgico ${ }^{(2)}$.

El conocimiento exhaustivo del problema que se va a enfrentar, la adecuada valoración y compensación preoperatoria del paciente, y la capacidad de adoptar medidas correctivas inmediatas en caso necesario, obligan al anestesiólogo a agudizar sus observaciones clínicas, apoyadas en el monitoreo específico, indispensable en este tipo de cirugía, con el fin de prever y minimizar la aparición de complicaciones. Aunque se habla de dificultades anestésicas de la cirugía laparoscópica, éstas no son puramente producidas por el manejo anestésico, sino que están relacionadas directamente con las particularidades de la técnica laparoscópica, pero las mismas deben ser diagnosticadas y tratadas precozmente por el anestesiólogo, con el objetivo de que ellas no repercutan grandemente en el estado del enfermo, con el propósito de disminuir la morbimortalidad quirúrgica.

Al tiempo que se da la anestesia para la colecistectomía videolaparoscópica, se originan condiciones especiales en la fisiología respiratoria con incremento de las presiones en la vía aérea y disminución de la adaptabilidad pulmonar ${ }^{(3-5)}$, imponiendo así la creación de cambios en los habituales modos de ventilar, referidos a la relación inspiración-espiración, en función de disminuir esas presiones, aumentar tal adaptabilidad y evitar la aparición de complicaciones procurando un éxito total de la intervención.

La fracción entre el tiempo inspiratorio y el espiratorio, nominada I:E, es determinada por la ventilación por minuto y la velocidad del flujo de aire; el lapso inspiratorio es determinado por el flujo y volumen tidal, el período espiratorio es determinado por el flujo de aire y su frecuencia ${ }^{(6)}$. La correlación I:E de 1:2 es el patrón común de la ventilación asistida a lo largo de la anestesia ${ }^{(7)}$. Velocidades lentas de flujo pueden reducir las presiones en la vías aéreas y mejorar la distribución de los gases por incremento del índice I:E ${ }^{(8,9)}$.

Sin embargo, buscando obtener los beneficios del tiempo inspiratorio prolongado, no siempre es necesario invertir completamente la correspondencia inspiración-espiración, lo que es un estímulo para utilizarla en otras situaciones de aireación no relacionadas con daño pulmonar, sino con necesidades de mejoría en el flujo de aire pulmonar en situaciones específicas durante la aireación mecánica transoperatoria, tal cual sucede en la colecistectomía videolaparoscópica ${ }^{6,7,10)}$.

El mecanismo de mejoría está relacionado con muchos factores, por ejemplo el incremento de la presión media en la vía aérea sin incremento en el valor pico, reclutamiento de alvéolos adicionales por enlentecimiento del flujo inspiratorio y desarrollo de un nivel positivo al final de la espiración (auto-PEEP o PEEP intrínseca). El alentamiento del flujo inspiratorio también podría disminuir el riesgo de volutrauma o barotrauma, los que desempeñan un rol en el fenómeno de lesión pulmonar asociada a la ventilación mecánica. Caballero y Camacho(11) señalaron que la estrategia que persigue incrementar el lapso inspiratorio, basada en el no incremento de la presión inspiratoria pico (PIP), será de mejores consecuencias fisiológicas. A pesar de esta mejoría en la aireación, puede incrementar los efectos nocivos colaterales cardiovasculares ${ }^{(12-15)}$.

Desde que el síndrome de dificultad respiratoria del adulto (SDRA) fuera descrito hace más de cuatro décadas por Ashbaugh, se han propuesto diferentes estrategias ventilatorias, a fin de restaurar la oxigenación arterial y la ventilación alveolar, y uno de los modos ventilatorios empleados es la inversión de la relación inspiración-espiración (IRI/E) ${ }^{(16)}$. Como táctica ventilatoria, el ajuste del tiempo inspiración-espiración se deberá considerar un mecanismo respiratorio en la aireación pulmonar protectora, que es capaz de mejorar la supervivencia considerando que se logra disminuir la presión en la vía aérea con altas posibilidades de aplicación en la ventilación asistida intraoperatoria para tales fines ${ }^{(17)}$.

\section{Cambios transoperatorios}

Cambios cardiovasculares. Las variaciones cardiovasculares se van a presentar fundamentalmente en el gasto cardíaco (GC), la presión en cuña pulmonar (PCP), la presión venosa central (PVC), la tensión arterial (PA) y la frecuencia cardíaca (FC), y van a depender directamente de su valor intraabdominal (PIA), del volumen de $\mathrm{CO}_{2}$ absorbido, de la posición de Trendelenburg, de la duración del procedimiento, de la ventilación y la técnica anestésica, de las enfermedades preexistentes y del volumen intravascular efectivo del paciente.

Con la posición de Trendelenburg y la elevación de la PIA, en pacientes ASA I y II se presenta un aumento inicial del retorno venoso y del gasto cardíaco, y prácticamente no se van a presentar mayores alteraciones que comprometan la 
hemodinámica, pero en la medida en que la PIA aumenta por encima de $15 \mathrm{mmHg}$ y la posición de Trendelenburg se hace mayor a $30^{\circ}$ con respecto a la horizontal, se va ocasionando un incremento en la presión de la vía aérea, de la intratorácica, la PVC, la tensión arterial, la resistencia venosa, y resistencia vascular sistémica; esto se explicaría por una compresión vascular tanto arterial como venosa, hipoxia e isquemia a nivel capilar y arteriolar, con liberación de péptidos que inhiben los reflejos tónicos vasculares locales y que a distancia se comportan como factores depresores del miocardio $^{(16-19)}$. $\mathrm{El} \mathrm{CO}_{2}$ puede producir arritmias, incremento en la concentración de catecolaminas circulantes, ascenso del gasto cardíaco, y pese a que la hipercapnia produce aumento de la resistencia vascular sistémica, si la acidosis respiratoria no se controla, podría terminar por producir un efecto neto de disminución de tal resistencia vascular sistémica. Cuando la PIA aumenta por encima de $30 \mathrm{mmHg}$ se va a encontrar una subida en la FC y disminución en los otros parámetros: PVC, GC y PA ${ }^{(20)}$.

Es interesante saber que, desde hace tres décadas, Kelman y colegas reportaban ya que a partir de una PIA de 22.5 mmHg se comenzaba a comprometer el gasto cardíaco, y que prácticamente después de $30 \mathrm{mmHg}$ tanto el GC como la PVC disminuían invariablemente ${ }^{(21)}$. Estudios realizados en personas sanas coinciden en el riesgo de comprometer el flujo esplácnico y la función renal (produciendo oliguria) cuando la PIA es mayor de $15 \mathrm{mmHg}^{(22)}$.

Reportados en el año 1994, los estudios de Feig y colaboradores utilizando neumoperitoneo con $15 \mathrm{mmHg}$ de PIA, en enfermos catalogados con ASA III y IV con alteraciones cardiorrespiratorias, destacaban un compromiso en el GC, a diferencia de lo que ocurría en los pacientes con ASA I y II ${ }^{(23)}$.

Cambios respiratorios. Las alteraciones a este campo van a depender, además de la PIA y del $\mathrm{CO}_{2}$ absorbido, de la edad, el peso, la función pulmonar preoperatoria, el grado de posición de Trendelenburg, la técnica anestésica, la duración del procedimiento y los agentes anestésicos. La posición forzada de Trendelenburg (mayor a 20 grados) va a disminuir la capacidad residual funcional, el volumen pulmonar total y el cumplimiento (compliance) pulmonar. Las variaciones en las propiedades pasivas mecánicas del pulmón y la reja torácica son reversibles y no entrañan mayor dificultad de manejo en individuos sanos. Si a pesar de esto hay evidencia de hipoxemia, hay que proceder a descartar, entre otros factores: condiciones preexistentes (obesidad mórbida), hipoventilación (posición forzada de Trendelenburg), derivación intrapulmonar (neumotórax), gasto cardíaco bajo (compresión de la vena cava inferior), o fallas técnicas del circuito o de la máquina de anestesia.

En el estudio citado antes, Feig ${ }^{(23)}$ también encontró en enfermos con ASA III y IV un gradiente $\mathrm{PaCO}_{2}-\mathrm{ETCO}_{2}$ de
$11 \mathrm{mmHg}$, el doble del valor encontrado en sujetos sanos, y unas presiones de inspiración-pico cinco a seis veces más altas que las halladas en personas saludables.

El neumoperitoneo con bióxido de carbono va a aumentar la $\mathrm{PaCO}_{2}(5-8 \mathrm{mmHg})$ y la concentración de $\mathrm{CO}_{2}(30 \%)$. En pacientes ASA III o IV, a pesar de que se aumenten los parámetros ventilatorios, incluso manteniendo un $\mathrm{CO}_{2}$ espirado normal, puede presentarse acidosis e hipercapnia refractaria, de ahí que en estos enfermos sea necesaria una adecuada valoración pulmonar preoperatoria $^{(24)}$.

En 1992, en un estudio de Puri y Singh, los enfermos intervenidos por cirugía laparoscópica con neumoperitoneo con bióxido de carbono, PIA de 12-14 mmHg, y posición de Trendelenburg forzada, lograban pasar de una concentración de $\mathrm{CO}_{2}$ de 216 a $282 \mathrm{~mL} / \mathrm{min}$ (un aumento de 30\%), en un tiempo de 35 minutos, incluso 10 minutos después de haber retornado a la posición normal $\left(0^{\circ}\right)$ y suspendido la PIA, indicando que ese gas continúa absorbiéndose después de haberse suspendido la insuflación peritoneal ${ }^{(25)}$.

\section{Complicaciones anestésico-quirúrgicas}

\section{Complicaciones transoperatorias relacionadas con el} neumoperitoneo. Las complicaciones de insuflar bióxido de carbono en la cavidad peritoneal podrían ser, desde menores, como el enfisema subcutáneo (cuando la aguja por donde se administra el gas queda en el tejido celular subcutáneo), hasta mayores, como un gran embolismo por $\mathrm{CO}_{2}$ (cuando la aguja queda erróneamente insertada en un vaso sanguíneo).

Las siguientes son las distintas posibilidades de enfrentar complicaciones cuando se utiliza dicho gas para el neumoperitoneo:
a. Enfisema subcutáneo
b. Neumotórax
c. Neumomediastino
d. Neumopericardio
e. Enfisema faríngeo
f. Explosiones
g. Producción de CO
h. Embolismo gaseoso
i. Hipercapnia marcada
j. Acidosis respiratoria severa
k. Compromiso de la RVS
1. Disminución del GC
m. Disminución de la PA
n. Arritmias

\section{Relación inspiración-espiración}

Por primera vez en 1971, el concepto de fase inspiratoria prolongada fue investigado por Reynolds, en neonatos con 
membrana hialina, y en 1976 Fuelihan demostró, en adultos con distrés respiratorio, el valor de la inversión del IE, cuya meta es mejorar el intercambio gaseoso sin contribuir a daño pulmonar ulterior ${ }^{(26)}$. La prolongación del tiempo inspiratorio redunda en una velocidad más baja del flujo inspiratorio, evitando vaivenes turbulentos que aumentan la resistencia en la vía aérea. La reversión del índice inspiración espiración puede generar beneficios en la oxigenación a través de cambios en las presiones de tal vía, que ocasionan reducción del espacio muerto y en el cortocircuito, a la vez que mejora la reciprocidad ventilación/perfusión. El indicador invertido en la correlación inspiración espiración podría ser aplicado con aireación volumétrica controlada, ventilación manométrica controlada y SIMW.

Inversión de la relación inspiración espiración con volumen control.

\section{Ventajas}

Garantiza un volumen de corriente definido.

Controla la onda de flujo inspiratorio.

Disponible en todos los ventiladores.

Existe una gran experiencia a nivel mundial y hace más gradual y fisiológica la transición de ventilación mecánica.

\section{Desventajas}

La presión inspiratoria máxima es variable y requiere monitoreo estricto y sedación profunda para evitar asincronía.

El objetivo de esta investigación es evaluar el comportamiento de las presiones intrapulmonares con la inversión del índice inspiración espiración en el transcurso de la colecistectomía electiva videolaparoscópica.

\section{MATERIAL Y MÉTODOS}

Se realizó un ensayo clínico controlado fase tres, en el período de septiembre de 2016 a febrero de 2020, para evaluar el comportamiento de dichas presiones intrapulmonares con la reversión del indicador inspiración espiración durante la colecistectomía electiva videolaparoscópica.

Se considera una población infinita con un nivel de confianza o seguridad de $95 \%$, predicción de $3 \%$, proporción $5 \%$ y una proporción esperada de pérdida de $15 \%$, la muestra estuvo formada por 106 pacientes, los cuales se dividieron en dos conjuntos de 53 elementos cada uno, por método aleatorio simple.

La fuente primaria de obtención de datos la constituyeron las historias clínicas y el resultado de un formulario. El procesamiento de la información obtenida se realizó mediante la utilización del programa SPSS con instalación de sistema Microsoft Windows 10.

\section{Criterios de inclusión:}

1. Pacientes atendidos en el hospital para colecistectomía electiva videolaparoscópica.

2. Con estatus ASA I, II, III.

3. Que den su disposición de participar en el estudio.

Criterios de exclusión:

1. Pacientes con ASA IV.

2. Quienes decidan retirarse del estudio.

3. Que se suspendan del acto operatorio.

Todos los pacientes se premedicaron con midazolam 0.05 $\mathrm{mg} / \mathrm{kg}$, luego se les preoxigenó con mascara facial de oxígeno al 100\%, la inducción se efectuó con midazolam 0.1-0.2 mg/ $\mathrm{kg}$, fentanyl 3-5 $\mu \mathrm{g} / \mathrm{kg}$, succinilcolina $1 \mathrm{mg} / \mathrm{kg}$ para facilitar la intubación; a continuación se realizó la intubación orotraqueal y se comprobó la correcta colocación del tubo endotraqueal, mediante la auscultación de ambos campos pulmonares y con la observación de la curva de bióxido de carbón espirado. La anestesia se mantuvo con fentanyl $0.5 \mu \mathrm{g} / \mathrm{kg}$ cada 30 minutos aproximadamente, midazolam acorde a necesidad, atracurio $0.1 \mathrm{mg} / \mathrm{kg}$ o vecuronio $0.01 \mathrm{mg} / \mathrm{kg}$, según requerimientos. Por vía inhalatoria se administró óxido nitroso para el mantenimiento, cuando no hubo contraindicación. No se utilizaron anestésicos volátiles.

Se monitorizaron frecuencia cardíaca, tensión arterial sistólica, tensión arterial diastólica, tensión arterial media, electrocardiograma de superficie en DII, saturación parcial de oxígeno, volumen por minuto, presión pico, valor meseta, $\mathrm{CO}_{2}$ espirado, frecuencia respiratoria de 12 por minuto y volumen corriente a $8 \mathrm{~mL} / \mathrm{kg}$, estos dos últimos parámetros se ajustaron buscando mantener el bióxido de carbono espirado entre 35-45 mmHg. Estas cifras se vigilaron cada cinco minutos antes, durante y después del neumoperitoneo.

Al finalizar el procedimiento se revirtieron los efectos del relajante muscular con neostigmina $0.04-0.07 \mathrm{mg} / \mathrm{kg}$ más atropina $0.01-0.02 \mathrm{mg} / \mathrm{kg}$, y el de la benzodiacepina con flumazenil $0.2 \mathrm{mg} / \mathrm{EV}$. Se extubó a los pacientes y se trasladaron a la sala de recuperación.

Grupo uno: tras la intubación, se acoplaron a la máquina de anestesia Fabius GS en modalidad volumen control, con relación inspiración-espiración 1:2 y luego del neumoperitoneo se invirtió el índice a 1.5:1.

Grupo dos: después de la intubación, se acoplaron a la máquina de anestesia Fabius GS en modalidad volumen control, con correlación inspiración-espiración 1:2.

El estudio se realizó en sujetos sometidos a colecistectomía quienes recibieron anestesia general. A los sujetos se les explicó el alcance del proyecto y firmaron el consentimiento 


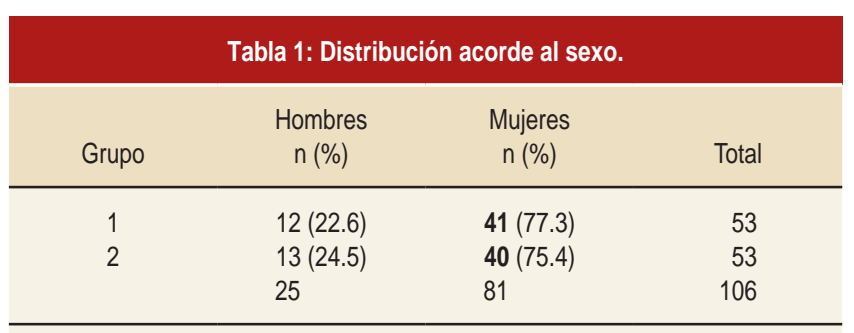

Fuente: Historia clínica.

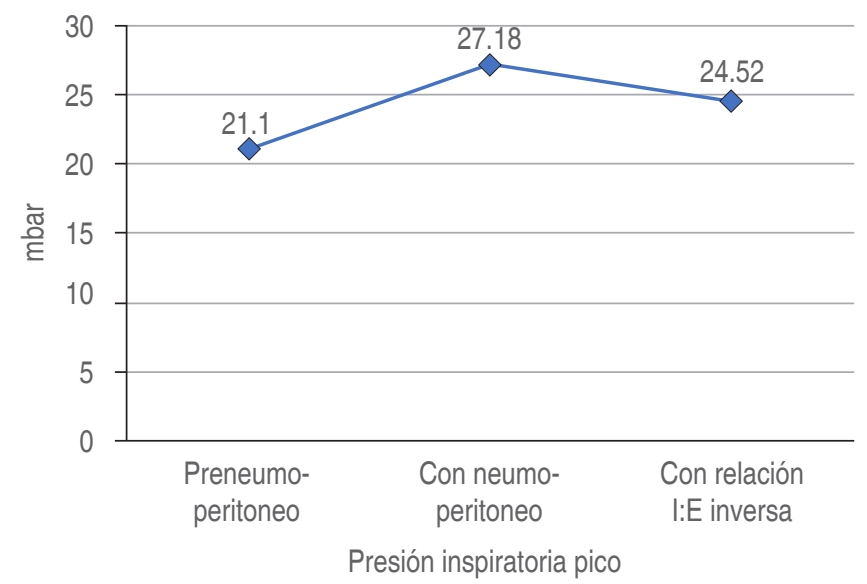

Figura 1: Comportamiento de la presión inspiratoria pico.

Fuente: Historia clínica.

informado. Para su realización, este proyecto se aprobó por el Comité de Ética del hospital.

\section{RESULTADOS}

En relación a la distribución según sexo, se observa un predominio de las mujeres en ambos grupos de estudio (Tabla 1).

En relación a la presión meseta, vemos un comportamiento similar al de la presión inspiratoria pico, observándose un aumento de la presión meseta luego del neumoperitoneo y una disminución de la misma luego de la inversión del índice inspiración-espiración (Figura 1).

En la Figura 2 se puede observar el comportamiento de la presión inspiratoria pico antes del neumoperitoneo, después del neumoperitoneo y luego de la inversión del índice inspiración-espiración, observándose un aumento de la presión pico luego del neumoperitoneo y una disminución significativa luego de la inversión del índice.

Sobre la distribución según alteraciones cardíacas encontramos que en el caso de las arritmias en el grupo 2 hubo una incidencia de $39.6 \%$ y en el grupo 1 de $34 \%$.

Relacionado a los cambios respiratorios, la diferencia mayor es a expensas de la hipercapnia con $45.3 \%$ en el grupo 1 y $62.3 \%$ en el grupo 2 (Tabla 2).

\section{DISCUSIÓN}

Los resultados obtenidos en este estudio, relacionados con la distribución del sexo, coinciden con lo planteado a nivel internacional: que el sexo femenino es el más afectado, con una proporción de 4.4 a 1.0, avalado por la fisiopatología de aquellas enfermedades relacionadas con la obesidad, multiparidad, uso de contraceptivos orales y otros ${ }^{(18)}$.

En relación con la edad, se observó que el mayor número de operados de ambos grupos se centraron entre 36-55 años, lo cual era esperado debido a que es en estas edades donde se concentra la mayor incidencia de patología de la vesícula biliar. Sin embargo, otros plantean que las complicaciones agudas de la vesícula son más frecuentes en los pacientes mayores de 60 años de edad, debido al mayor tiempo de evolución de la enfermedad ${ }^{(27)}$.

Según los expertos convocados, existe escasa literatura sobre el tema de la inversión de la relación inspiración espiración en anestesia, aunque en su investigación, Smith RP y Fletcher $\mathrm{R}^{(28)}$ demostraron que en el postoperatorio de la cirugía cardíaca obtuvieron valores más bajos de presiones intrapulmonares con relación a la ventilación convencional. Empero, en su trabajo de inversión en enfermos neuroquirúrgicos, Clarke no encontró diferencias significativas en las presiones intrapulmonares ${ }^{(29)}$. Un estudio realizado en

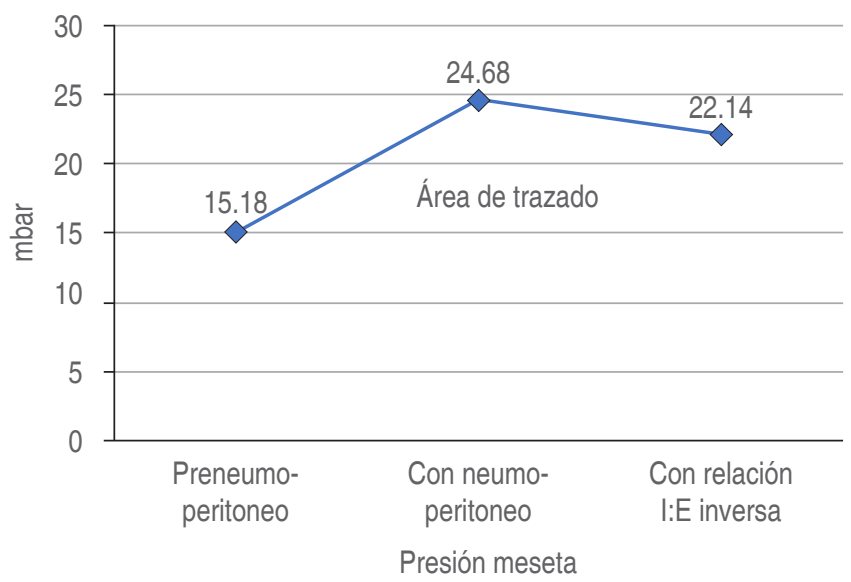

Figura 2: Comportamiento de la presión meseta.

Fuente: Historia clínica.

Tabla 2: Distribución según cambios respiratorios.

\begin{tabular}{ccc} 
Grupo & $\begin{array}{c}\text { Hipercapnia } \\
\mathrm{n}(\%)\end{array}$ & $\begin{array}{c}\text { Hipocapnia } \\
\mathrm{n}(\%)\end{array}$ \\
\hline 1 & $24(45.3)$ & $2(3.8)$ \\
2 & $33(62.3)$ & $3(5.7)$ \\
\hline
\end{tabular}

Fuente: Historia clínica. 
el Hospital Universitario de Cartagena, Colombia, reveló mejores niveles de presion en la vía aérea y una mejoría significativa en la distensibilidad pulmonar con mejores parámetros de oxigenación en 15 pacientes ingresados en la UCI con SDRA ${ }^{(30)}$.

Al buscar alteraciones cardíacas, en la distribución de los grupos se encontró esa incidencia, la cual estando cerca de $25 \%{ }^{(31)}$, se considera similar pero con un ligero incremento a la encontrada en otros trabajos; al tener en cuenta que la causa de las arritmias puede ser la insuflación del $\mathrm{CO}_{2}$ en la cavidad abdominal, entonces se piensa que la técnica ventilatoria usada en el conjunto dos pudo haber tenido algún efecto en la aparición de esta complicación, debido a la mayor hipercapnia en este grupo. No obstante, en opinión de Fernando Chirio ${ }^{(32)}$ las arritmias son fácilmente controladas a través de un correcto ajuste de la aireación pulmonar.

La absorción sistémica de $\mathrm{CO}_{2}$ produce hipercarbia y acidosis, libera catecolaminas y estimula al sistema nervioso central (SNC), que produce un cronotropismo e inotropismo positivos y origina taquicardia sinusal y extrasístoles ventriculares. Por otro lado, las bradiarritmias, que incluyen bradicardia sinusal, ritmo nodal e incluso asistolia, se atribuyen a una respuesta vagal secundaria a distensión abdominal e irritación peritoneal por la insuflación.

Hay que señalar que ciertos trabajos revisados coinciden en afirmar que la gran variabilidad de los protocolos anestésicos y sobre todo de las técnicas de ventilación han constituido un obstáculo para la comparación de los resultados expuestos en las distintas series estudiadas ${ }^{(33)}$.

En la comparación de la incidencia de hipotensión, en el grupo uno hubo $7.5 \%$ y en el grupo dos fue de 5.7\%. Esta variable no tiene diferencias importantes entre ellos, por lo que se infiere que la ocurrencia de la disminución de la tensión arterial está en estrecha relación con el neumoperitoneo y no con la técnica ventilatoria. No obstante, la literatura refiere que no importa si ocurre hipertensión o hipotensión, siempre habrá una reducción del índice cardíaco ${ }^{(34)}$.
La hipertensión arterial fue otra de las variables estudiadas y sin embargo no tuvo diferencias importantes entre los grupos, el uno con $39.6 \%$ y el dos con $32.1 \%$. Se considera que el aumento de la tensión arterial media y resistencias vasculares sistémicas aparentemente es independiente de la técnica ventilatoria, porque ocurre en ambos conjuntos con similar comportamiento. En definitiva, esto se ha referido con semejantes características en la bibliografía consultada. El uso de óxido nitroso tiene efectos análogos al bióxido de carbono, causando hipertensión arterial, según datos reportados por Cristchley y asociados ${ }^{(35)}$. El incremento de la presión arterial también puede atribuirse a la elevación reflejo de las resistencias vasculares sistémicas en respuesta a la distensión abdominal.

En conexión con los cambios respiratorios, la literatura revisada concuerda en que el neumoperitoneo, por razones obvias, aumenta la $\mathrm{PaCO}_{2}$ a partir de los cinco minutos de insuflado el gas ${ }^{(3,5)}$. Es uno de los cambios más comunes durante la cirugía laparoscópica. Esto se debe a un aumento de $\mathrm{CO}_{2}$ por absorción subcutánea o transperitoneal del $\mathrm{CO}_{2}$ de la insuflación. La magnitud está en relación con la solubilidad del gas, la PIA y la duración de la distensión abdominal, en particular cuando existe patología pulmonar, y hay elevación en el espacio muerto ventilatorio, reducción del movimiento del diafragma y disminución de la eliminación pulmonar del gas. El monitoreo continuo de la $\mathrm{PetCO}_{2}$ detecta oportunamente la hipercapnia y, de ser necesario, la gasometría arterial seriada corrobora esta condición. Una insuflación lenta previene dicha hipercapnia. La prolongación del tiempo inspiratorio produce una velocidad más lenta de flujo inspiratorio.

\section{CONCLUSIÓN}

Los autores consideran que la inversión de la inspiración espiración contribuyó a mejorar los efectos del neumoperitoneo sobre las presiones intrapulmonares al lograr su disminución, manteniendo una oxigenación adecuada y nulos efectos cardiovasculares.

\section{REFERENCIAS}

1. Kim MS, Soh S, Kim SY, Song MS, Park JH. Comparisons of pressurecontrolled ventilation with volume guarantee and volume-controlled 1:1 equal ratio ventilation on oxygenation and respiratory mechanics during robot-assisted laparoscopic radical prostatectomy: a randomizedcontrolled trial. Int J Med Sci. 2018;15:1522-1529.

2. Góis CJR, De Oliveira IR, Passos LM, Alves JA. Transumbilical videolaparoscopic (single site) liver biopsy with laparoscopy equipment. J Minim Access Surg. 2016;12:135-138.

3. Kitamura H, Tsuji T, Yamamoto D, Takahashi T, Kadoya S, Kurokawa M, Bando H. Efficiency of fluorescent cholangiography during laparoscopic cholecystectomy for subvesical bile ducts: a case report. Int J Surg Case Rep. 2019;57:194-196.

4. Schraibman V, Epstein MG, Naman MG, de Vasconcellos MAL. Singleport robotic cholecystectomy. Initial and pioneer experience in Brazil. Einstein (Sao Paulo) 2015;13:607-610.
5. Ozdemir-van Brunschot DM, van Laarhoven KC, Scheffer GJ, Pouwels $\mathrm{S}$, Wever KE, Warlé MC. What is the evidence for the use of low-pressure pneumoperitoneum? A systematic review. Surg Endosc. 2016;30:2049-2065.

6. Jin Ha Park, In Kyeong Park, Seung Ho Choi, Darhae Eum, Min-Soo Kim. Volume-controlled versus dual-controlled ventilation during robotassisted laparoscopic prostatectomy with steep Trendelenburg position: a randomized-controlled trial. J Clin Med. 2019;8:2032.

7. Kim KN, Kim DW, Jeong MA, Sin YH, Lee SK. Comparison of pressure-controlled ventilation with volume-controlled ventilation during one-lung ventilation: a systematic review and meta-analysis. BMC Anesthesiol. 2016;16:72.

8. Gad M, Gaballa K, Abdallah A, Abdelkhalek M, Zayed A, Nabil H. Pressure-controlled ventilation with volume guarantee compared to volume-controlled ventilation with equal ratio in obese patients undergoing laparoscopic hysterectomy. Anesth Essays Res. 2019;13:347-353. 
9. Kim MS, Soh S, Kim SY, Song MS, Park JH. Comparisons of pressurecontrolled ventilation with volume guarantee and volume-controlled 1:1 equal ratio ventilation on oxygenation and respiratory mechanics during robot-assisted laparoscopic radical prostatectomy: a randomizedcontrolled trial. Int J Med Sci. 2018;15:1522-1529.

10. Jaju R, Jaju PB, Dubey M, Mohammad S, Bhargava AK. Comparison of volume controlled ventilation and pressure controlled ventilation in patients undergoing robot-assisted pelvic surgeries: an open-label trial. Indian J Anaesth. 2017;61:17-23.

11. Caballero LA, Camacho AV. Modos de ventilación. En: Caballero LA. ed. Terapia Intensiva. Terapia Intensiva TII, Ciudad de La Habana, ECIMED; 2008. pp. 486-532.

12. Jing Tan, Zhenghuan Song, Qingming Bian, Pengyi Li, Lianbing Gu. Effects of volume-controlled ventilation vs pressure-controlled ventilation on respiratory function and inflammatory factors in patients undergoing video-assisted thoracoscopic radical resection of pulmonary carcinoma. J Thorac Dis. 2018;10:1483-1489.

13. Ball L, Costantino F, Fiorito M, Amodio S, Pelosi P. Respiratory mechanics during general anaesthesia. Ann Transl Med. 2018;6:379.

14. Guay J, Ochroch EA, Kopp S. Intraoperative use of low volume ventilation to decrease postoperative mortality, mechanical ventilation, lengths of stay and lung injury in adults without acute lung injury. Cochrane Database Syst Rev. 2018;2018:CD011151.

15. Jin Ha Park, Jong Seok Lee, Jae Hoon Lee, Seokyung Shin, Nar Hyun Min, Min-Soo Kim. Effect of the prolonged inspiratory to expiratory ratio on oxygenation and respiratory mechanics during surgical procedures. Medicine (Baltimore). 2016;95:e3269.

16. Federica Lovisari, Gergely H. Fodor, Ferenc Peták, Walid Habre, Sam Bayat. Effect of PEEP and I:E ratio on cerebral oxygenation in ARDS: an experimental study in anesthetized rabbit. BMC Anesthesiol. 2019;19:110.

17. Regli A, Pelosi P, Malbrain MLNG. Malbrain. Ventilation in patients with intra-abdominal hypertension: what every critical care physician needs to know. Ann Intensive Care. 2019;9:52.

18. Kyung Mi Kim, Jung Ju Choi, Dongchul Lee, Wol Seon Jung, Su Bin Kim, Hyun Jeong Kwak. Effects of ventilatory strategy on arterial oxygenation and respiratory mechanics in overweight and obese patients undergoing posterior spine surgery. Sci Rep. 2019;9:16638.

19. Youn Yi Jo, Hyun-Jeong Kwak. What is the proper ventilation strategy during laparoscopic surgery? Korean J Anesthesiol. 2017;70:596-600.

20. Jain SV, Kollisch-Singule M, Sadowitz B, Dombert L, Satalin J, Andrews $\mathrm{P}$, Gatto LA, et al. The 30-year evolution of airway pressure release ventilation (APRV). Intensive Care Med Exp. 2016;4:11.
21. Kelman GR, Swapp GH, Smith I, Benzie R, Nanette LM. Cardiac output and arterial blood-gas tensión during laparoscopy. Brit J Anaesth. 1972;44:155-161.

22. Souza GMC, Ganni Mara S Santos, Fabiano T Barbosa, Tamara Melnik. Intraoperative ventilation strategies for obese patients undergoing bariatric surgery. Cochrane Database Syst Rev. 2018;2018:CD011758.

23. Feig BW, Berger DH, Dougherty TB, Dupuis JF, Hsi B, Hickey RC, Ota DM. Pharmacologic intervention can reestablish baseline hemodynamic parameters during laparoscopy. Surgery. 1994;733-739.

24. García-de-la-Asunción J, García-del-Olmo E, Galan G, Guijarro R, Martí F, Badenes R, Perez-Griera J, et al. Glutathione oxidation correlates with one-lung ventilation time and $\mathrm{PO}_{2} / \mathrm{FiO}_{2}$ ratio during pulmonary lobectomy Redox Rep. 2016;21:219-226.

25. Puri GD, Singh H. Ventilatory effects of laparoscopy under general anaesthesia. Br, J Anaesth. 1992;68:211-213.

26. Fuleihan SF, Wilson RS, Pontoppidan H. Effect of mechanical ventilation with end-inspiratory pause on blood-gas exchange. Anesth Analg. 1976;55:122-130.

27. Vinueza AM, et al. Anestesia para colecistectomía laparoscópica. Experiencia en 200 casos. Rev Ecu Anest. 1999;3:39-42.

28. Smith RP, Fletcher R. Pressure-controlled inverse ratio ventilation after cardiac surgery. Eur J Anaesthesiol. 2011;18:106-109.

29. Clarke JP. The effects of inverse ratio ventilation on intracranial pressure: a preliminary report. Intensive Care Med. 1997;23:106-109.

30. Dueñas CR, García CE, Carvajal M, Martínez A, Alvarado D, Julio L, et al. Respuesta hemodinámica y ventilatoria a la prolongación del tiempo inspiratorio controlado por presión vs controlado por volumen en pacientes con SDRA. Acta Colombiana de Cuidado Intensivo. 2002;5:12-18

31. Smith RP, Fletcher R. Pressure-controlled inverse ratio ventilation after cardiac surgery. Eur J Anaesthesiol. 2011;18:106-109.

32. Chirio F. Anestesia en cirugía laparoscópica. Rev Argent Anestiol. 1996;54:400-419.

33. Costa SGM, Santos GM, Zimpel SA, Melnik T. Intraoperative ventilation strategies for obese patients undergoing bariatric surgery: systematic review and meta-analysis. BMC Anesthesiol. 2020;20:36.

34. Scott TE, Das A, Haque M, Bates DG, Hardman JG. Management of primary blast lung injury: a comparison of airway pressure release versus low tidal volume ventilation. Intensive Care Med Exp. 2020;8:26.

35. Critchley LAH, Critchley JAJH, Gin T. Haemodynamic changes in patients undergoing laparoscopic cholecystectomy: measurement by transthoracic electrical bioimpedance. Br J Anesth 1993;70:681-683. 